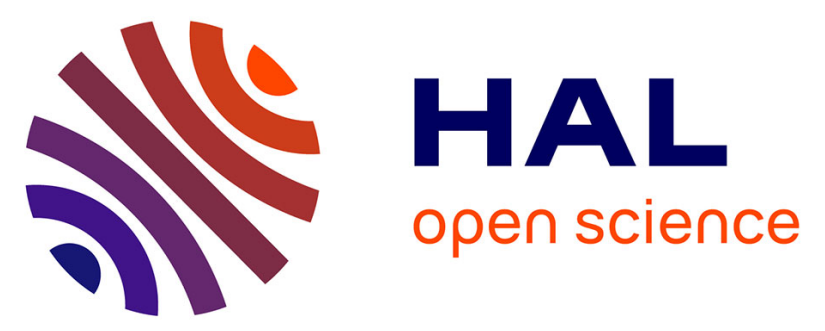

\title{
Effets positifs d'une exposition à des séquences vidéo ralenties sur l'attention, la communication sociale et les troubles du comportement chez 4 enfants autistes sévères: une étude translationnelle pilote
}

Eve Meiss, Carole Tardif, Thomas Arciszewski, Bruno Dauvier, Bruno Gepner

\section{To cite this version:}

Eve Meiss, Carole Tardif, Thomas Arciszewski, Bruno Dauvier, Bruno Gepner. Effets positifs d'une exposition à des séquences vidéo ralenties sur l'attention, la communication sociale et les troubles du comportement chez 4 enfants autistes sévères: une étude translationnelle pilote. Neuropsychiatrie de l'Enfance et de l'Adolescence, 2015. hal-01787262

\author{
HAL Id: hal-01787262 \\ https://hal.science/hal-01787262
}

Submitted on 7 May 2018

HAL is a multi-disciplinary open access archive for the deposit and dissemination of scientific research documents, whether they are published or not. The documents may come from teaching and research institutions in France or abroad, or from public or private research centers.
L'archive ouverte pluridisciplinaire HAL, est destinée au dépôt et à la diffusion de documents scientifiques de niveau recherche, publiés ou non, émanant des établissements d'enseignement et de recherche français ou étrangers, des laboratoires publics ou privés. 
Effets positifs d'une exposition à des séquences vidéo ralenties sur l'attention, la communication sociale et les troubles du comportement chez 4 enfants autistes sévères: une étude translationnelle pilote

Positive impact of slowed down video sequences on attention, social communication and behaviour in four children with severe autism: a pilot translational study

E. Meiss ${ }^{1}$, C. Tardif ${ }^{2}$, T. Arciszewski ${ }^{2}$, B. Dauvier ${ }^{2}$, B. Gepner ${ }^{1}$

${ }^{1}$ Laboratoire NICN (Neurobiologie des Interactions Cellulaires et Neuropathologie), UMR CNRS 7259, Faculté de Médecine Marseille Nord, Aix-Marseille Université

${ }^{2}$ Laboratoire PSYCLE (Psychologie de la Cognition, du Langage et de l'Emotion), Aix-Marseille Université, Maison de la Recherche, 29 avenue Robert Schuman, 13100 Aix-en-Provence

\footnotetext{
${ }^{\circ}$ Auteur correspondant.

Email : bruno.gepner@univ-amu.fr (B. Gepner)

Conflit d'intérêt : aucun.
} 


\section{Résumé}

L'une des théories psychopathologiques développementales des troubles de la communication et des interactions sociales dans l'autisme propose que les informations de l'environnement sont souvent trop rapides pour être traitées en temps réel par les personnes avec autisme. Quelques études expérimentales ont montré qu'un ralentissement des informations sonores et visuelles entraîne de meilleures performances dans des tâches imitatives, verbales et cognitives chez des enfants autistes, notamment ceux atteints d'autisme modéré à sévère. Ces recherches ont abouti au développement de Logiral ${ }^{\mathrm{TM}}$, une application numérique de ralentissement simultané des informations audio-visuelles, sans altération de la fréquence acoustique des sons.

Dans la présente étude, nous avons présenté sur ordinateur, en vitesse ralentie grâce à Logiral $^{\mathrm{TM}}$, des séquences audio-visuelles de personnes exprimant diverses émotions faciales ou différentes consignes, à quatre enfants autistes sévèrement atteints pris en charge en hôpital de jour, au cours de 10 séances de 30 à 45 minutes sur 3 mois. Nous avons mesuré avec des échelles les fréquences d'apparition d'indices comportementaux tels que l'attention, la communication verbale, et non-verbale, la réciprocité des échanges, et les comportements inappropriés, puis analysé leurs variations en fonction des séances.

Nos résultats révèlent, chez les quatre enfants, une augmentation significative de la fréquence de l'attention, de la communication non-verbale et de la réciprocité des échanges, mais pas d'augmentation significative de la communication verbale, et une diminution significative des comportements inappropriés, au cours du temps.

Les résultats de cette première étude translationnelle ouverte sont encourageants, puisque l'exposition à des stimuli présentés au ralenti améliore des comportements clés pour la communication sociale. D'autres études impliquant des sujets et des tâches contrôles sont nécessaires pour déterminer l'intérêt et les limites du ralentissement comme nouveau principe thérapeutique dans les troubles du spectre de l'autisme.

MOTS CLES : Autisme - Etude de cas - Ralentissement - Recherche translationnelle Communication - Attention - Interaction - Traitement perceptif.

\section{Abstract}

Among theoretical explanations of the typical deficits in social interaction and verbal and non-verbal communication in autism, one suggests that environmental information in the visual and auditory modalities may change too fast to be processed on-time by persons with autism. It has been shown that some children with autism are impaired in perceiving and integrating physical and biological movements, and categorizing ambiguous phonemes, when acoustic and visual signals at displayed at real-time speed. Other studies have also shown that slowing down audio-visual information enhances verbal, cognitive and imitative abilities in some autistic children, especially those with moderate to severe autism. On this basis, we developed a numeric application aimed at slowing down synchronously visual and auditory cues without impairing acoustic frequency (Logiral ${ }^{\mathrm{TM}}$ ).

In this study, four children having severe autism were presented various audiovisual sequences such as verbal instructions or adults exhibiting several facial emotions, displayed at slowed down speeds via Logiral ${ }^{\mathrm{TM}}$ on a PC. Participants were tested over 10 sessions during 3 months in their child day-care unit. The experimenter assessed the occurrence of 'positive' (attention, verbal communication, non-verbal communication, social reciprocity) and negative (inappropriate) behavioral cues, and variations of these cues were compared across the sessions.

Our results reveal a significant increase of positive behaviours (attention, non-verbal communication and social reciprocity) over time, but no significant increase of verbal communication, and a significant decrease of inappropriate behaviours, in the four children tested.

These results overall argue for a benefit of slowing down audiovisual stimuli on social communication in these four children. This first open pilot study is encouraging. Further 
studies involving control tasks and control subjects are necessary to identify benefits and limits of slowness as a new therapeutic tool for children having autism spectrum disorders.

KEYWORDS: Autism - Case study - Slow down - Translational research - Communication Attention - Social interaction - Perceptual processing. 
Depuis la première description de l'autisme infantile par Kanner en 1943 [1], ce syndrome a été progressivement étendu à un ensemble relativement hétérogène de manifestations cliniques appelées troubles envahissants du développement (TED), caractérisés par des déficits plus ou moins sévères et envahissants de la communication verbale et non verbale et des interactions sociales, et par des intérêts atypiques et des comportements restreints et répétitifs $[2,3]$.

Plusieurs théories sensorielles, cognitives ou socio-émotionnelles ont été proposées depuis 40 ans pour expliquer ces difficultés et particularités développementales et comportementales, dans l'autisme, en particulier celles s'appuyant sur un déficit de la reconnaissance et de l'appariement trans-modal des indices émotionnels contenus dans les mouvements du visage et du corps, et la voix [4], des déficits complexes de l'imitation [5], des troubles du fonctionnement exécutif (inhibition, anticipation, planification) [6], un déficit de perception et de compréhension des états mentaux d'autrui via l'observation de visages, de scènes sociales ou de scénarios sollicitant normalement l'empathie (déficit de construction d'une théorie de l'esprit, déficit d'empathisation, avec a contrario excès de systématisation) [7], une faiblesse de la cohérence centrale (difficulté à grouper les percepts en un tout cohérent et unifié) [8], un sur-fonctionnement perceptif (visuel et auditif) et intellectuel dans certains domaines de prédilection [9].

Notre théorie psychopathologique développementale de l'autisme, en partie compatible avec l'ensemble de ces théories, s'appuie quant à elle sur l'existence de déficits du traitement perceptif et intégratif des informations environnementales de fréquences temporelles élevées, qu'il s'agisse du mouvement visuel [10] ou encore des sons de la parole et des informations proprioceptives [11]. Autrement dit, les informations en provenance de l'environnement humain et non-humain sont trop rapidement mouvantes ou changeantes pour être traitées en temps réel, de manière simultanée, globale et cohérente, par de nombreuses personnes autistes. Par exemple les signaux audio-visuels émis par un interlocuteur et a fortiori plusieurs interlocuteurs lors d'une interaction sociale impliquant sans cesse de multiples flux de paroles, de gestes, d'expressions faciales et de mouvements corporels rapides, complexes, et difficilement prévisibles seraient soit évités du fait de leurs charges sensorielle et informationnelle excessives, soit traités plus lentement, générant des troubles de la compréhension des signaux de communication verbale, faciale, émotionnelle, et une désynchronisation dans les échanges avec l'interlocuteur.

Parallèlement, les personnes autistes développent très précocement des capacités parfois hors du commun (en intensité, rapidité, efficacité) pour détecter, observer, percevoir et mémoriser des détails informationnels multiples notamment les détails visuels ou sonores (biais local et sur-fonctionnement perceptif [9]). Cette focalisation de leur attention sur les détails prend du temps, se fait plus ou moins au détriment d'une perception globale, mais elle peut aussi aboutir à des performances accrues voire de véritables prouesses mnésiques pour les dates, les lettres, les mots, les langues, ou pour les chiffres et le calcul (rôle conjoint des synesthésies ou confusions sensorielles). Ces prouesses qui sont souvent peu en phase avec les exigences socio-adaptatives de la société, peuvent cependant être créatives et conférer à certains un avantage cognitif et/ou évolutif (en mathématiques et en informatique par exemple) par rapport aux personnes non autistes.

Ce puzzle de déficits et de lenteur pour traiter les informations dynamiques rapides, et de surfonctionnement et de rapidité pour traiter les détails a abouti au concept de Désordres $d u$ Traitement Temporo-Spatial (DTTS) des stimuli multi-sensoriels [11] qui pourrait être au cœur des principaux troubles moteurs, cognitifs, socio-émotionnels et comportementaux de nombreuses personnes atteintes de troubles du spectre de l'autisme.

Plusieurs études expérimentales semblent confirmer cette théorie, en montrant le bénéfice pour certains enfants autistes d'une présentation ralentie des signaux audio-visuels. En effet, la présentation ralentie d'expressions faciales émotionnelles et non-émotionnelles permet de mieux identifier l'expression qui leur est présentée, et induit davantage d'imitation immédiate $[12,13]$. Un ralentissement des mouvements faciaux et gestes corporels entraîne une 
augmentation de la production de comportements d'imitation de ces mouvements et gestes par certains enfants [14]. Enfin, le ralentissement de certains phonèmes améliore la catégorisation de ceux-ci [15], et le ralentissement de consignes verbales simples et complexes en améliore la compréhension [16]. En contexte expérimental, le ralentissement via un ordinateur des signaux visuels et sonores semble donc accroître et améliorer la perception visuelle et auditive et leur association bimodale chez les enfants autistes, et plus particulièrement chez ceux ayant un degré relativement sévère d'autisme et/ou un faible niveau développemental verbal. Le monde va trop vite pour de nombreux enfants autistes [17] mais aussi pour nombre d'adultes autistes [18], et le ralentissement (naturel ou artificiel) de l'environnement pourrait les aider à mieux le percevoir et le décoder.

Dans la continuité de ces travaux, l'application numérique Logiral ${ }^{\mathrm{TM}}[19,20]$ a été développée pour permettre de ralentir de façon synchrone le son et l'image de séquences présentées à l'écran d'un ordinateur sans déformation de la fréquence acoustique du son, c'est-à-dire sans modification de la tonalité de la voix.

Dans la présente recherche, nous testons la plateforme Logiral ${ }^{\mathrm{TM}}$ auprès de quatre enfants autistes afin de déterminer si l'utilisation régulière du ralentissement des signaux audiovisuels permet à ces enfants d'acquérir de nouvelles habilités de compréhension, de communication et d'expression verbale et socio-émotionnelle, ainsi qu'une amélioration des échanges interpersonnels. Nous faisons l'hypothèse que le ralentissement des stimuli visuels et auditifs pourrait favoriser la compréhension verbale de ces enfants, augmenter leur engagement attentionnel sur les supports audio-visuels proposés, favoriser l'imitation spontanée et les interactions sociales ainsi que la communication verbale et non verbale.

\section{METHODE}

\section{Participants}

Quatre enfants autistes, diagnostiqués selon les critères de la CIM-10 [2], ont été recrutés pour participer à cette étude, après information complète et loyale de leurs parents, et obtention de leur signature d'un formulaire de consentement libre et éclairé, incluant pour tous l'autorisation de les filmer pendant les séances, et pour trois d'entre eux, l'autorisation de diffuser ces films à des fins d'enseignement, de formation et de recherche.

Ces enfants sont régulièrement pris en charge dans un hôpital de jour à Marseille. Ils ont également tous été évalués avec la CARS (échelle d'évaluation de l'autisme infantile) [21] et l'ECSP (échelle de communication sociale précoce) [22].

\section{Participant $1:$ E.}

E. est une petite fille, non verbale, âgée de 6 ans et 11 mois au début de notre étude. Les supports audio-visuels lui ont été présentés à une vitesse de ralentissement de $70 \%$ (par rapport à la vitesse temps-réel qui est de 100\%), soit un degré de ralentissement considéré comme correct pour elle, c'est-à-dire suscitant une attention optimale de sa part à l'égard des supports audio-visuels.

Ses caractéristiques d'âge, de diagnostic, de sévérité des troubles, de niveau de communication, sont détaillées (Tableau 1).

E. est une enfant repliée sur elle-même qui n'échange pas avec les autres enfants et qui ne sollicite l'adulte qu'en cas de besoin (besoins fondamentaux ou de contact). Elle ne communique pas par la parole mais par des sons et des gestes; elle produit très fréquemment des sons stéréotypés et des activités motrices répétitives, ainsi que des comportements particuliers centrés sur son corps. Elle ne montre pas d'intérêt pour les jouets et les objets mais sait néanmoins les utiliser de manière appropriée en fonction de ses besoins. Elle peut réagir vivement à la frustration par des pleurs et être violente envers elle-même mais jamais envers les autres. Anxieuse, elle présente des difficultés à focaliser son attention sur une tâche, montre des réactions particulières à certains sons, elle porte également de l'intérêt aux odeurs 
et peut sélectionner des objets en fonction de sensations qu'elle trouve agréables ou non. E. met du temps à réagir et à intégrer ce qui se passe dans son environnement. Elle ne fait pas l'effort de faire des expériences nouvelles dont elle pourrait tirer profit, chaque comportement doit d'abord être encouragé par l'adulte, souvent avec insistance, sinon elle reste fixée sur des rituels. Elle ne va ensuite faire les choses que si elle y voit un intérêt, si elle ressent un besoin ou une gêne.

\section{Participant $2: K$.}

$\mathrm{K}$. est un petit garçon, non verbal, âgé de 6 ans et 9 mois au début de notre étude. Les supports audio-visuels lui ont été présentés à une vitesse de ralentissement de $80 \%$.

Ses caractéristiques d'âge, de diagnostic, de sévérité des troubles, de niveau de communication, sont détaillées (Tableau 1).

K. est né prématurément à 6 mois de grossesse. Il a été nourri par sonde jusqu'à ses 4 mois. Ce petit garçon préfère être seul mais il lui arrive d'échanger avec les adultes et les autres enfants, et il se sert fréquemment de l'adulte pour obtenir quelque chose. Il ne communique pas par la parole mais d'abord par le geste puis par les sons. C'est un enfant très expressif qui comprend et réagit rapidement aux gestes ou aux consignes verbales simples même en l'absence de contexte. K. est souvent agité, il présente des activités motrices répétitives très fréquentes et réagit très vivement à la frustration par des cris, des pleurs ou la violence. Son utilisation des objets est très ritualisée : il ne joue pas de manière appropriée avec les jouets mais utilise les objets de manière globalement appropriée. Il est capable d'imitation gestuelle avec et sans objet. C'est un enfant taquin qui aime le jeu et l'échange s'il est dans un cadre particulier comme des activités en petit groupe ou lorsqu'il est seul avec peu d'adultes. Il aime les sensations, être porté, serré, faire des roulades sans interruption, il porte beaucoup d'intérêt à la télévision, aux images, aux sons et à la musique.

\section{Participant 3 : J.}

J. est un petit garçon, non verbal, âgé de 6 ans et 8 mois au début de notre étude. Les supports audio-visuels lui ont été présentés à une vitesse de ralentissement de $65 \%$.

Ses caractéristiques d'âge, de diagnostic, de sévérité des troubles, de niveau de communication, sont détaillées (Tableau 1).

J. est un enfant solitaire qui répond peu aux sollicitations. Il n'échange pas avec les autres enfants mais il sait solliciter l'adulte par le geste lorsqu'il en a besoin. Son visage reflète ses émotions, bien qu'elles soient peu variées car J. est passif et angoissé, il pleure beaucoup à l'Hôpital de Jour car la séparation avec sa maman est difficile. En revanche, il peut être volontaire, souriant, en lien avec les autres et très présent durant les activités ou les soins à l'Hôpital de Jour. Il présente des difficultés attentionnelles et une faible notion de son corps selon le cadre dans lequel il se trouve, surtout lorsqu'il n'a pas de repères. Il joue peu avec des objets ou des jouets, il a surtout tendance à les agripper et ne les utilise pas toujours de manière appropriée. J. a tendance à se boucher les oreilles lorsqu'un bruit est intense, il ne présente pas de gestes ni de sons stéréotypés. Il peut mettre du temps à réagir selon le contexte : lorsqu'il est dans un cadre calme et sécurisant, J. intègre très bien les informations.

\section{Participant $4: V$.}

$\mathrm{V}$. est un petit garçon, non verbal, âgé de 7 ans et 5 mois au début de notre étude. Les supports audio-visuels lui ont été présentés à une vitesse de ralentissement de $80 \%$.

Ses caractéristiques d'âge, de diagnostic, de sévérité des troubles, de niveau de communication, sont détaillées (Tableau 1).

V. n'interagit pas avec les autres enfants et reste principalement seul, mais il communique de façon non verbale avec l'adulte en fonctions de ses besoins. Son temps de réaction augmente en fonction de ses envies, du nombre d'enfants autour de lui ou si son environnement n'est pas assez sécurisant. Il peut être attentif aux mouvements faciaux lorsqu'on lui épelle des lettres par exemple qu'il tente d'imiter en différé. V. joue particulièrement avec des jouets musicaux qu'il colle à son oreille ou avec lesquels il fait des "expériences". Les sons et la musique 
attirent fortement son attention, il aime se créer des sensations sonores, se balancer à leur écoute et il produit souvent des sons stéréotypés. Son utilisation des objets est très ritualisée. $\mathrm{V}$. a fréquemment des difficultés attentionnelles et porte une attention exagérée à certaines choses. Il réagit vivement à la frustration et il peut, dans ces cas-là, être violent envers son entourage. C'est un enfant réactif qui comprend les consignes qu'on peut lui énoncer. Il montre aussi un grand d'intérêt pour les chiffres et les lettres qu'il sait reconnaître.

\section{Protocole expérimental}

Afin de pouvoir présenter aux participants diverses séquences vidéo dans plusieurs vitesses, nous avons utilisé l'application numérique appelée Logiral ${ }^{\mathrm{TM}}$ (acronyme de LOGIciel de RALentissement) qui permet de ralentir le son et l'image d'une vidéo de façon synchrone, sans altérer la fréquence acoustique et donc la tonalité des sons et de la voix [19,20]. Cette application numérique a été installée sur un ordinateur PC portable (Dell Latitude ${ }^{\circledR}$ ).

Chaque enfant a participé à 10 séances d'environ 30 minutes, soit une séance par semaine durant 3 mois. Les jours et horaires étaient réguliers. Ces séances ont été mises en place sous la forme d'un atelier expérimental régulier adapté au niveau de développement et aux intérêts de chaque enfant. Ces séances se sont déroulées dans une pièce dédiée où se trouvait une table sur laquelle étaient posés l'ordinateur, la caméra destinée à filmer le déroulement des séances ainsi que les différents matériels nécessaires selon les séances. L'enfant et l'expérimentateur étaient assis à la table côte à côte.

Durant chaque séance, l'enfant était sollicité par l'expérimentateur pour observer des séquences vidéo au ralenti, puis réagir par pointage aux questions posées sur les vidéos qu'il venait de visionner ou bien aux consignes reçues durant la vidéo. Deux séances d'essais préliminaires ont précédé le démarrage de l'étude proprement dite, afin de familiariser les enfants avec les différents matériels, et déterminer le ralentissement optimal pour chaque enfant. Un ralentissement optimal signifie celui qui suscite le maximum d'attention sur une scène visuelle connue de l'enfant (un dessin animé qu'il regarde à domicile).

Durant chaque séance, trois types de tâches ont été proposées à partir des stimuli présentés à l'écran de l'ordinateur en vitesse ralentie:

1) Une tâche de reconnaissance de 6 expressions faciales émotionnelles de personnes familières ou non familières. L'enfant devait ensuite répondre à des questions par pointage : devant lui étaient disposées 2 photographies de ces mêmes émotions, il devait alors reconnaître et pointer l'émotion visionnée auparavant parmi ces 2 photographies.

2) Une tâche de compréhension de consignes à partir de supports vidéo où l'expérimentateur énonce des consignes d'imitation de gestes simples, ou prononce des consignes d'actions simples ou complexes portant sur différents matériels, consignes que l'enfant doit ensuite réaliser. D'autres consignes étaient issues des films de l'instituteur spécialisé montrant les illustrations d'un livre: 2 images extraites du livre étaient ensuite disposées devant l'enfant et celui-ci devait reconnaître et désigner l'image demandée.

3) Une tâche de questions sur des dessins animés nécessitant une réponse par pointage des personnages sur des images extraites de ces dessins animés.

\section{Recueil des données}

Les résultats de chaque enfant sont évalués en fonction de la fréquence des comportements observés durant les séances. Pour ce faire, 2 grilles de cotation spécifique ont été remplies immédiatement au terme de chacune des séances par l'expérimentateur:

1) Une grille de comportements dits "positifs" évalués selon un score qui peut varier de 0 à 4 , 0 correspondant à une fréquence d'apparition nulle d'un comportement et 4 correspondant à une apparition très fréquente. Dix-huit comportements sont ainsi codés et regroupés en 4 catégories : réciprocité des échanges, attention, communication verbale et communication non verbale (Annexe 1). La moyenne des scores, et donc de la fréquence d'observation des 
comportements composant chacune des 4 catégories, est ensuite calculée afin d'obtenir le score de chaque catégorie par séance. Ce sont ces moyennes que nous analysons par la suite.

2) Une grille de cotation de comportements inappropriés ou troubles du comportement, permettant un codage de la fréquence de leur apparition d'après un score allant de 0 à 4 (0 correspondant à une fréquence nulle et 4 correspondant à une apparition très fréquente de ces comportements) (Annexe 2). La durée des comportements inadaptés est également codée selon un score qui varie de 0 à 4 (0 équivaut à une durée nulle, c'est-à-dire une absence de comportement inapproprié, et 4 correspond à une durée longue).

Des enregistrements filmés de toutes les séances ont permis d'ajuster après-coup les cotations réalisées pendant ou à la fin de chaque séance.

\section{RESULTATS}

\section{Analyse des données}

Nous analysons en premier lieu l'évolution globale des comportements positifs au cours du temps en prenant comme mesure à expliquer le score moyen issu du codage des comportements positifs. Ces comportements sont dans un second temps dissociés dans les 4 catégories précédemment évoquées (attention, communication verbale, communication nonverbale, réciprocité des échanges). Nous comparons ensuite les évolutions de ces 4 catégories afin de préciser l'évolution de ces comportements au cours des séances, et savoir si l'un ou plusieurs de ces comportements bénéficie plus que les autres des séances. Enfin, l'évolution au cours des séances des comportements inappropriés est analysée de manière séparée.

Ces données de mesures répétées au cours des séances sont traitées au moyen d'un modèle linéaire généralisé à effet mixte (GLMM : Generalized Linear Mixed Model) [23,24]. Les analyses sont réalisées à l'aide du logiciel de statistiques $\mathrm{R}$ [25] et de la librairie lme4 [26]. Ces GLMM permettent l'analyse de l'effet d'une variable temporelle (les séances) en interaction avec une variable catégorielle (le type d'indice). Il est ainsi possible de comparer les évolutions respectives des différents indices tout en considérant la structure hiérarchique des données due au fait que chaque observation est liée à l'un des 4 sujets. La variable Sujet est donc également intégrée dans le modèle sous la forme d'un effet aléatoire (1|Sujet) qui représente la variabilité interindividuelle. La démarche se fait par comparaison de modèles successifs. Après le calcul d'un modèle de référence, ou modèle nul, ici constitué par la variable Sujet seule, toute variable ajoutée qui améliorera l'ajustement du modèle pourra être considérée comme apportant une contribution significative.

\section{Résultats concernant les comportements "positifs"}

Quatre modèles ont successivement été estimés puis comparés les uns aux autres par ordre de complexité croissante (Tableau 2). L'indice AIC - Akaike Information Criterion [27] - donne une indication globale de la qualité du modèle. Il considère simultanément l'ajustement et la parcimonie du modèle. Plus l'AIC est bas, meilleur est le modèle, ce qui permet d'identifier rapidement le meilleur modèle. Par ailleurs, la comparaison deux à deux de modèles emboités permet d'obtenir une probabilité associée au gain d'ajustement. Le modèle $\mathrm{m} 1$ (score seance $+(1 \mid$ sujet $))$ ajoute au modèle nul individuel $(\mathrm{m} 0:$ score $\sim(1 \mid$ sujet $)$ l'effet des séances. M2 est un modèle additif avec les indices et la séance (deux paramètres sont évalués indépendamment - score $\sim$ seance +indice $+(1$ |sujet) et le troisième est un modèle avec un terme d'interaction entre les variables "séance" et "indice" (score $\sim$ seance*indice + (1|sujet). Ce modèle est particulièrement intéressant dans le cas de cette étude puisqu'il fait la supposition que les différents indices varient de manière contrastée en fonction des séances. La comparaison des modèles par les AIC (AIC (M0)=485.85; $\mathrm{AIC}(\mathrm{M} 1)=442.73$; $\mathrm{AIC}(\mathrm{M} 2)=283.40$; $\mathrm{AIC}(\mathrm{M} 3)=245.75)$ et les probabilités associées à la comparaison des modèle deux à deux montre que le meilleur modèle est le dernier modèle qui comporte le terme d'interaction (Tableau 2). Ce résultat suggère que le travail au cours des séances ne produit pas le même effet sur les différents comportements étudiés ici. 
La Figure 1 montre des évolutions positive claires et homogènes de l'attention, de la réciprocité des échanges et de la communication non verbale chez les 4 enfants. La communication verbale en revanche ne parait pas s'améliorer au cours des séances. Afin d'analyser plus précisément ces évolutions, nous avons estimé les paramètres du modèle $\mathrm{m} 3$ par la procédure GEE (Generalized Estimating Equation [28], librairie geepack [29]). Ce type d'analyse permet d'estimer chaque paramètre individuellement et d'y associer une probabilité. Les traitements d'un GEE prennent pour référence une des modalités de la variable catégorielle à traiter, dans notre cas, la modalité communication verbale retenue pour sa stabilité. Les autres comportements (communication non verbale, attention et réciprocité) lui sont donc comparés. Le modèle dans son ensemble rend bien compte des données observées $\left(\mathrm{R}^{2}=0.79\right)$. Plus précisément, les résultats montrent en premier lieu que la pente pour la communication verbale n'est pas significativement différente de 0 (séance: Com.Verb. $=0,02 ; \mathrm{p}=0,1)$, ce qui confirme sa stabilité à un niveau bas tout au long des séances. En revanche, les pentes associées aux trois autres comportements sont significativement plus élevées (Séance: Attention $=0,2, \quad \mathrm{p}<0,01$; Séance: Com.Non.Verb. $=0,19, \quad \mathrm{p}<0,01$; Séance: Réciprocité $=0,23, \mathrm{p}<0,01)$. L'attention, la communication non verbale, et la réciprocité montrent donc des évolutions positives et significatives au cours des séances chez les 4 enfants.

\section{Résultats concernant les comportements inappropriés}

L'indicateur utilisé pour analyser les comportements inappropriés est constitué par l'addition de la fréquence de ces comportements (score allant de 0 à 4 ) et de leur durée (score qui varie de 0 à 4). Plus cet indicateur est élevé plus les comportements inappropriés ont été importants dans le cours de la séance. Cela permet d'étudier l'évolution d'une seule variable "comportements inappropriés".

L'effet global de la succession des séances sur l'importance quantitative des comportements inappropriés est étudié à l'aide d'un modèle linéaire estimé par GEE (modèle : comportements inappropriés séance). En moyenne, les comportements inappropriés diminuent significativement au cours du temps (séance $=-2.189, \mathrm{SE}=1.029, \mathrm{p}<.05, \mathrm{R}^{2}=$ .27) chez les 4 enfants, ce qui témoigne donc d'une amélioration globale du comportement au cours des 10 séances réalisées.

L'évolution des comportements inappropriés de chaque enfant au cours des 10 séances est représentée graphiquement sur la Figure 2: une baisse régulière apparait pour deux des 4 enfants et de manière encore plus marquée pour $\mathrm{V}$.

\section{DISCUSSION}

Cette première étude ouverte translationnelle pilote visait à tester auprès de 4 enfants autistes sévères l'impact thérapeutique du ralentissement des signaux audio-visuels sur leur attention, leur communication et leurs interactions sociales, ainsi que sur leurs comportements inappropriés. Nos résultats mettent en évidence une augmentation significative de l'attention pour les supports audio-visuels proposés, de la réciprocité des échanges avec l'expérimentateur, et de la communication non verbale, chez les 4 enfants au cours du temps. En revanche, les séances effectuées n'ont pas eu d'effet significatif sur leur communication verbale.

Nos résultats montrent également une diminution significative des comportements inappropriés chez les 4 enfants au cours du temps.

Cependant, nos résultats ne permettent pas d'affirmer que les effets bénéfiques ne sont dus qu'à l'exposition répétée des enfants à des stimuli audio-visuels ralentis. Des effets de familiarité avec l'expérimentateur, générant un meilleur ajustement réciproque de l'expérimentateur et des enfants, et des effets d'apprentissage concourent sans doute également à l'évolution positive des enfants au cours des séances. Pour pouvoir être plus affirmatif quant à un effet spécifique du ralenti, il faudrait tester un petit groupe contrôle (ne disposant pas du ralenti au cours des séances), ou faire, au sein de ce groupe d'enfants, une comparaison entre 
des séances effectuées au ralenti et d'autres séances présentées en vitesse temps-réel. Mais étant donnée la sévérité clinique et le très faible niveau développemental des 4 enfants participants, et compte tenu du temps et du nombre de séances très limités pour effectuer une telle comparaison, nous avons fait le choix méthodologique de mener une étude pilote ouverte. Nos résultats ne sont donc évidemment pas généralisables à l'ensemble de la population autistique. Il est également difficile d'établir un protocole absolument rigoureux et systématique d'une séance à l'autre, et d'appliquer strictement un même protocole pour tous les enfants, car il est important, particulièrement avec ces enfants sévèrement atteints, de considérer à chaque séance la disponibilité, la motivation, l'humeur, le niveau de fatigue, les capacités et les difficultés de chaque enfant. Ceci implique donc une adaptation des supports.

Il semblerait dès lors intéressant de poursuivre l'entraînement des enfants au ralenti sur une durée plus importante et en intégrant de nouveaux supports afin d'élargir les possibilités de travail à davantage de capacités cognitives. Peu de mesures d'apprentissage ont pu être effectuées au cours de ces 3 mois d'étude du fait du faible niveau développemental des enfants. En effet, aucun d'entre eux n'a développé de capacités de pointage par exemple, d'où la faiblesse des réponses aux questions posées et la difficulté de mesurer leur niveau de compréhension des séquences visionnées. Sur la base de nos résultats, la poursuite des séances pourrait leur être profitable en termes d'acquisition de nouvelles capacités de communication.

Les résultats de cette étude pilote translationnelle sont donc encourageants. Il est nécessaire de mener des études supplémentaires impliquant des sujets et des conditions contrôles afin de mesurer l'utilité et les limites du ralentissement comme nouveau dispositif thérapeutique dans les troubles du spectre de l'autisme.

Remerciements : Nous remercions chaleureusement les parents des 4 enfants inclus dans l'étude, ainsi que toute l'équipe de l'hôpital de jour de Plombières à Marseille (14 ${ }^{\text {ème }}$ Arrondissement, Association Régionale pour l'Intégration, direction Dr. François Soumille).

\section{BIBLIOGRAPHIE}

1. Kanner, L. (1943). Autistic disturbances of affective contact. Nervous Child, 2, 217-250.

2. Organisation Mondiale de la Santé (OMS) (1993). Classification Internationale des Maladies et des problèmes de santé connexes, CIM-10/ICD 10. Paris, Masson.

3. American Psychiatric Association (2000). Diagnostic and statistical manual of mental disorders (4th ed., text rev.). Washington, DC: Author.

4. Hobson, R.P., Ouston, J., \& Lee, A. (1988). What's in a face? The case of autism. British Journal of Psychology, 79, 441-453.

5. Nadel, J. (2003). Imitation et autisme. Cerveau \& Psycho, 4.

6. Hill, E.L. (2004). Executive dysfunction in autism. Trends in Cognitive Sciences, 8, 26-32.

7. Baron-Cohen, S., Knickmeyer, R.C., Belmonte, M.K. (2005). Sex differences in the brain: implications for explaining autism. Science, 310, 819-823.

8. Frith, U. (1989). Autism: explaining the enigma. Basic Blackwell.

9. Mottron, L. (2004). L'autisme: une autre intelligence. Sprimont: Mardaga.

10. Gepner, B., Mestre, D. (2002). Rapid visual-motion integration deficit in autism. Trends in Cognitive Sciences, 6, 455.

11. Gepner, B. \& Féron, F. (2009). Autism: a world changing too fast for a mis-wired brain ? Neuroscience and Biobehavioral Reviews, 33, 1227-1242.

12. Tardif, C., Lainé, F., Rodriguez, M., \& Gepner, B. (2007). Slowing down presentation of facial movements and vocal sounds enhances facial expression recognition and induces facial-vocal imitation in children with autism. Journal of Autism and Developmental Disorders, 37, 1469-1484.

13. Lainé, F., Tardif, C., \& Gepner, B. (2008). Amélioration de la reconnaissance et de l'imitation d'expressions faciales chez des enfants autistes grâce à une présentation visuelle et sonore ralentie. Annales Médico-Psychologiques, 166, 533-538.

14. Lainé, F., Rauzy,S., Tardif, C., \& Gepner, B. (2010). Slowing down the presentation of facial and body movements enhances imitation performance in children with severe autism. Journal of Autism and Developmental Disorders, 41, 983-996.

15. Tardif, C., Thomas, K., Gepner, B., \& Rey, V. (2002). Contribution à l'évaluation du système phonologique explicite chez des enfants autistes. Parole, 21, 35-72. 
16. Lainé, F., Rauzy, S., Gepner, B., \& Tardif, C. (2009). Prise en compte des difficultés de traitement des informations visuelles et auditives rapides dans le cadre de l'évaluation diagnostique de l'autisme. Enfance $n^{\circ} 1,133-141$.

17. Gepner, B., Tardif, C. (2009). Le monde va trop vite pour les autistes. La Recherche, 436, 56-59.

18. Gepner B. (2014). Autismes. Ralentir le monde extérieur, calmer le monde intérieur. Paris : Odile Jacob.

19. Tardif, C., Gepner, B. (2012). Logiral [Logiciel PC]. Téléchargeable sur http://centrepsycle-amu.fr/logiral/

20. Tardif, C., Gepner, B. (2014). Logiral [Application IOS et Android]. Paris: Auticiel.

21. Schopler, E., Reichler, R. J., DeVellis, R. F., \& Daly, K. (1980). Toward objective classification of childhood autism: Childhood Autism Rating Scale (CARS). Journal of Autism and Developmental Disorders, 10, 91-103.

22. Guidetti M., Tourrette C. (2009). Echelle de communication sociale précoce. Eurotests Edition.

23. Lindsey, J.K. (2000). Applying Generalized Linear Models,Springer-Verlag: New York Inc.

24. Noël, Y. (2013). Psychologie statistique avec R. Springer Verlag : France.

25. R Core Team (2013). R: A language and environment for statisticalcomputing. R Foundation for Statistical Computing, Vienna, Austria.URL http://www.R-project.org/

26. Bates D., Maechler M., Bolker B., Walker S. (2013). lme4: Linear mixed-effects models using Eigen and S4. R package version 1.0-5. http://CRAN.R-project.org/package=lme4

27. Akaike, H. (1974). A new look at the statistical model identification. Automatic Control, IEEE Transactions, 19(6), 716-723.

28. Gardiner, J. C., Luo, Z., \& Roman, L. A. (2009). Fixed effects, random effects and GEE: What are the differences? Statistics in Medicine, 28, 221-239. doi:10.1002/sim.3478

29. Halekoh, U., Højsgaard, S., \& Yan, J. (2006). The R package geepack for generalized estimating equations. Journal of Statistical Software, 15(2), 1-11.

30. Wilkinson GN, Rogers CE. (1973). Symbolic Description of Factorial Models for Analysis of Variance. Journal of the Royal Statistical Society Series C (Applied Statistics). 1973;22(3):392-399.

Tableau 1. Résumé des caractéristiques cliniques des 4 enfants

\begin{tabular}{|c|c|c|c|c|}
\hline & E. & $\mathbf{K}$. & $\mathbf{J}$. & V. \\
\hline Genre & Féminin & Masculin & Masculin & Masculin \\
\hline $\begin{array}{c}\text { Âge au début de } \\
\text { l'étude }\end{array}$ & 6 ans et 11 mois & 6 ans et 9 mois & 6 ans et 8 mois & 7 ans et 5 mois \\
\hline $\begin{array}{c}\text { Diagnostic } \\
\text { principal selon la } \\
\text { CIM-10 }\end{array}$ & $\begin{array}{l}\text { F } 84.0 \\
\text { Autisme infantile }\end{array}$ & $\begin{array}{c}\text { F } 84.0 \\
\text { Autisme infantile }\end{array}$ & $\begin{array}{c}\text { F } 84.0 \\
\text { Autisme infantile }\end{array}$ & $\begin{array}{l}\text { F } 84.0 \\
\text { Autisme infantile }\end{array}$ \\
\hline $\begin{array}{l}\text { Diagnostic } 2 \text { selon } \\
\text { la CIM-10 }\end{array}$ & $\begin{array}{c}\text { F 71.1 } \\
\text { Retard mental } \\
\text { moyen avec } \\
\text { déficience du } \\
\text { comportement } \\
\text { nécessitant une } \\
\text { surveillance ou un } \\
\text { traitement }\end{array}$ & $\begin{array}{c}\text { F 71.1 } \\
\text { Retard mental } \\
\text { moyen avec } \\
\text { déficience du } \\
\text { comportement } \\
\text { nécessitant une } \\
\text { surveillance ou un } \\
\text { traitement }\end{array}$ & $\begin{array}{c}\text { F 71.1 } \\
\text { Retard mental } \\
\text { moyen avec } \\
\text { déficience du } \\
\text { comportement } \\
\text { nécessitant une } \\
\text { surveillance ou un } \\
\text { traitement }\end{array}$ & $\begin{array}{c}\text { F } 71.1 \\
\text { Retard mental } \\
\text { moyen avec } \\
\text { déficience du } \\
\text { comportement } \\
\text { nécessitant une } \\
\text { surveillance ou un } \\
\text { traitement }\end{array}$ \\
\hline Score CARS* & $\begin{array}{l}44,5 \\
\text { Autisme sévère }\end{array}$ & $\begin{array}{l}44 \\
\text { Autisme sévère }\end{array}$ & $\begin{array}{l}42,5 \\
\text { Autisme sévère }\end{array}$ & $\begin{array}{l}46,5 \\
\text { Autisme sévère }\end{array}$ \\
\hline $\begin{array}{c}\text { Niveau socio- } \\
\text { communicatif à } \\
\text { l'ECSP** }\end{array}$ & 10 mois & 15 mois & 10 mois & 11 mois \\
\hline
\end{tabular}

*CARS : Childhood Autism Rating Scale.

**ECSP : Echelle de communication sociale précoce. 
Tableau 2. Comparaison et résumé des Modèles Linéaires Généralisés à effet Mixte (GLMM) testés.

\begin{tabular}{|c|c|c|c|}
\hline & Signification du modèle & AIC & $\begin{array}{c}\text { Probabilité que le modèle } \\
\text { soit meilleur que le } \\
\text { précédent }\end{array}$ \\
\hline $\begin{array}{c}\text { Modèle M0 } \\
\text { score (1|sujet })\end{array}$ & $\begin{array}{l}\text { Variance interindividuelle } \\
\text { mais pas d'évolution } \\
\text { temporelle }\end{array}$ & 485,85 & \\
\hline $\begin{array}{c}\text { Modèle m1 : } \\
\text { score } \sim \text { seance }+(1 \mid \text { sujet })\end{array}$ & $\begin{array}{l}\text { Variance interindividuelle } \\
\text { + évolution temporelle } \\
\text { similaire sur les } 4 \text { indices }\end{array}$ & 442.76 & $\mathrm{P}<.001$ \\
\hline $\begin{array}{c}\text { Modèle m2 : } \\
\text { score } \sim \text { seance+indice }+(1 \mid \text { sujet })\end{array}$ & $\begin{array}{l}\text { Variance interindividuelle } \\
+ \text { différence de niveau } \\
\text { moyen en fonction des } \\
\text { indices }\end{array}$ & 283.40 & $\mathrm{P}<.001$ \\
\hline $\begin{array}{c}\text { Modèle m3 : } \\
\text { score } \sim \text { seance*indice+(1|sujet })\end{array}$ & $\begin{array}{c}\text { Variance } \\
\text { interindividuelle+ } \\
\text { évolution temporelle } \\
\text { différente en fonction des } \\
\text { indices }\end{array}$ & 245.75 & $\mathrm{P}<.001$ \\
\hline
\end{tabular}

Note : la notation est conforme à celle proposée par Wilkinson et Rogers (1973) [30], telle qu'utilisée dans R. 
Figure 1 : Évolution des scores d'attention, de communication non-verbale, de communication verbale, et de réciprocité des échanges, obtenus par chacun des 4 enfants au cours des 10 séances.

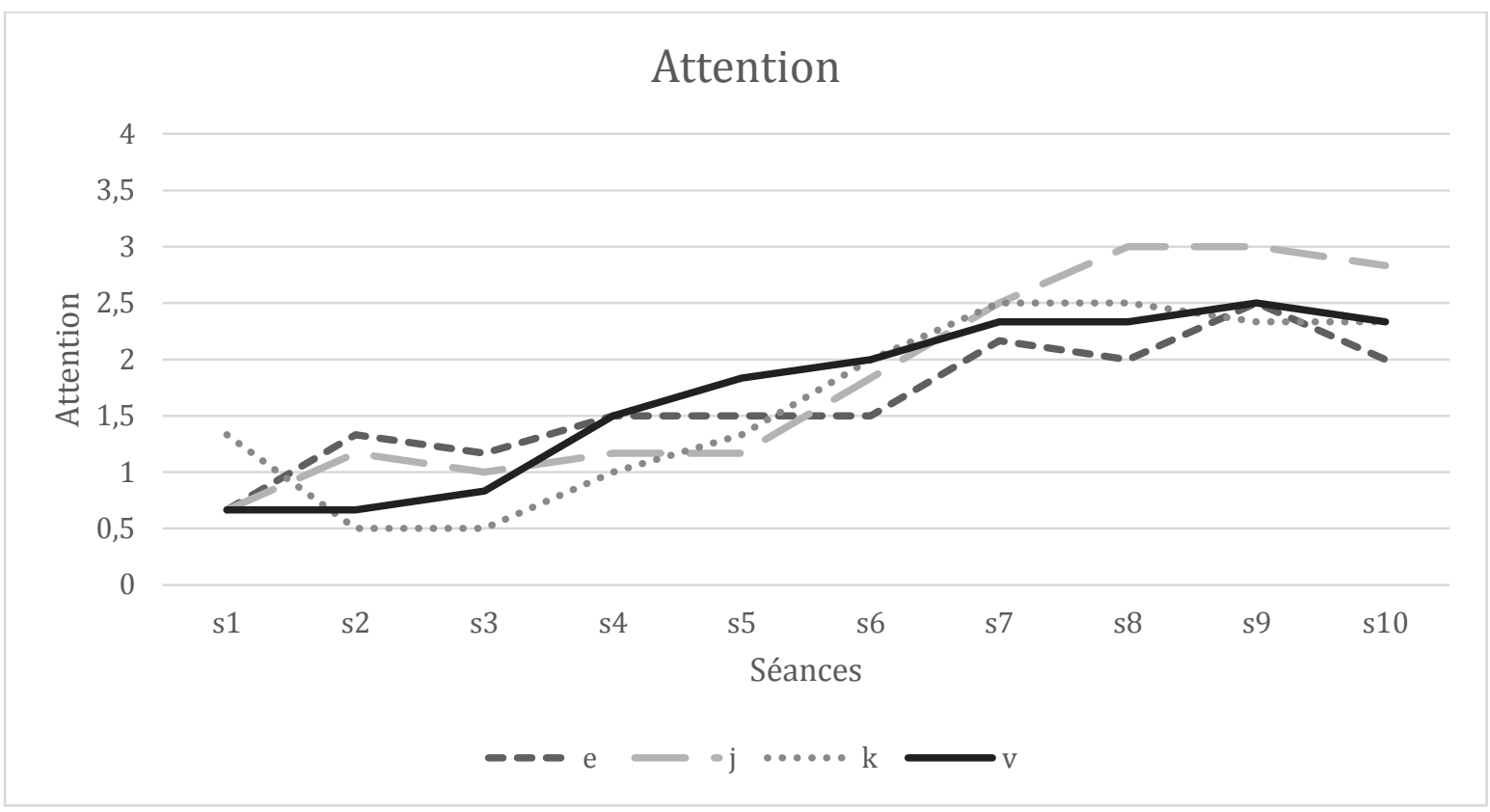

\section{Communication non verbale}

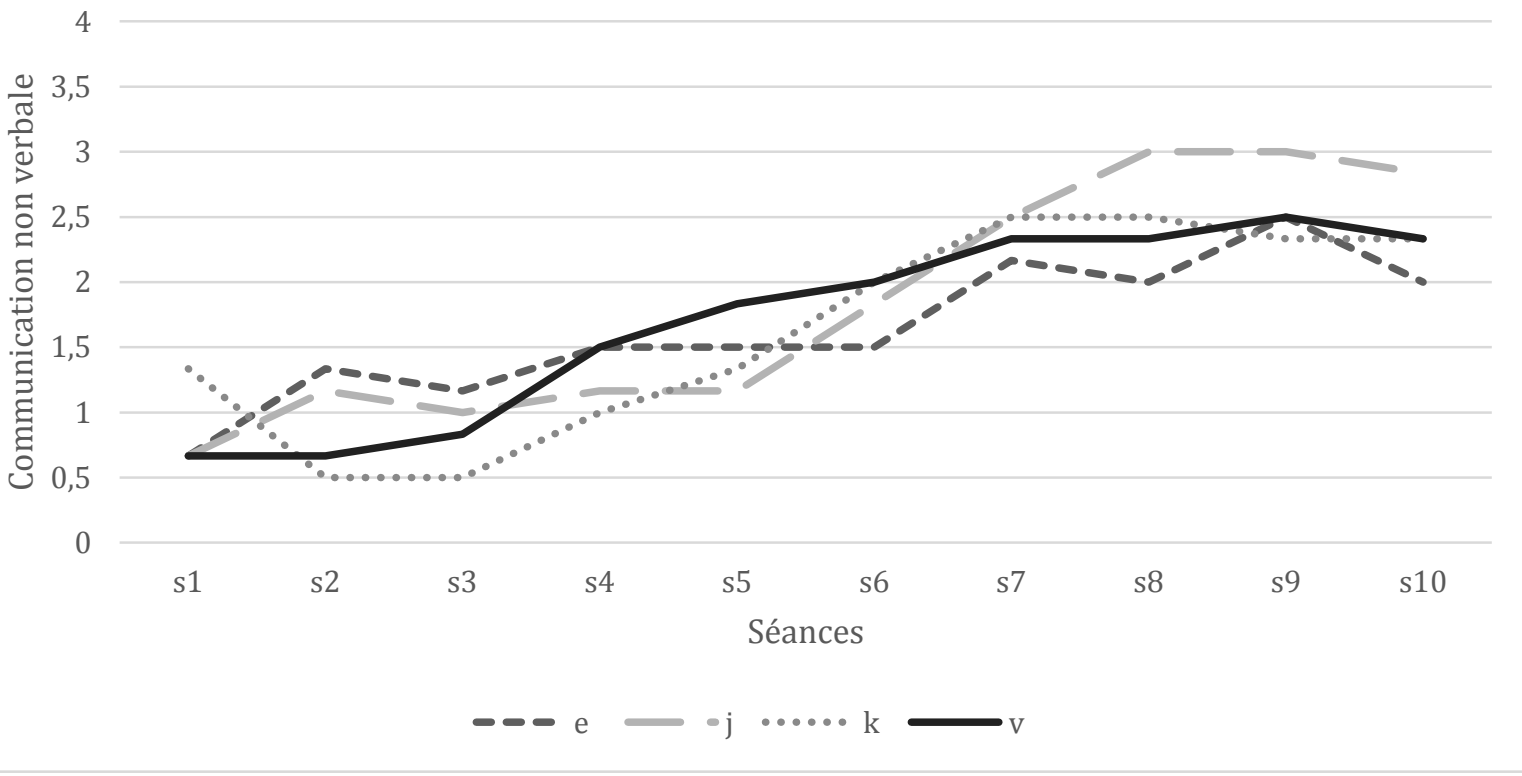




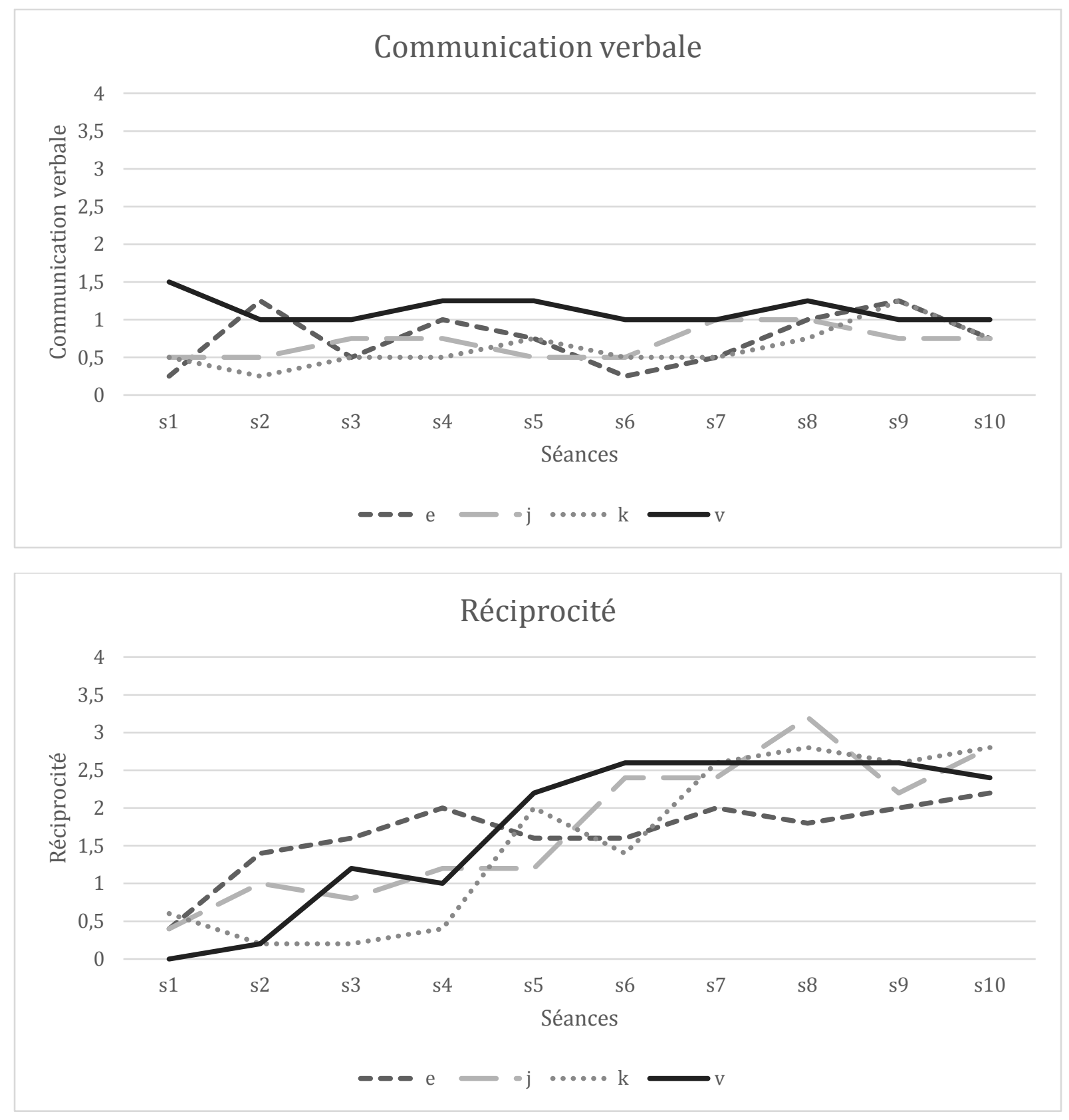


Annexe 1 : Comportements « positifs »
A) Attention
1-Attention visuelle
2- Attention auditive
3- Attention conjointe
B) Communication verbale
4- Compréhension du langage oral
5- Echolalies (immédiates ou différées)
6- Imitations vocales (sons, mots, phrases...)
7- Utilisation d'un langage adapté
C) Communication non verbale
8-Compréhension des gestes communicatifs
9- Compréhension des expressions émotionnelles
10- Imitations gestuelles
11- Echanges de regards
12- Production de gestes communicatifs (Pointage...)
13- Expressions émotionnelles
D) Réciprocité des échanges
14- Participation / Engagement dans la séance
15- Réponses immédiates (aux questions / sollicitations)
16- Réponses différées
17- Initiatives spontanées
18- Maintien des échanges

\section{Annexe 2 : Comportements inappropriés}

1- Stéréotypies perturbant la séance

2- Autostimulations perturbant la séance

3- Ne reste pas à sa place lors des activités

4- Rituels gênants lors de la séance

5- Auto-agressivité (violence dirigée contre soi)

6- Hétéro-agressivité (violence sur autrui)

7- Colère

8- Violence sur objet

9- Cris

10- Agitation

11- Utilisation inadéquate des objets

12- Intolérance au changement

13- Intolérance à la frustration

14- Intolérance au bruit et/ou au mouvement

15- Repli sur soi 